\title{
Adaptive Control for the Stabilization and Synchronization of N onlinear Gyroscopes
}

\author{
B. A. Idowu ${ }^{\mathrm{a}, ~}{ }^{*}$ RongweiGuo ${ }^{\mathrm{b},}$ U. E. Vincent ${ }^{\mathrm{c}, \mathrm{d}, *}$ \\ ${ }^{\mathrm{a}}$ Department of Physics, Lagos State University, Ojo, Nigeria. ${ }^{\mathrm{b}}$ School of Mathematics \\ and Physics, Shandong Institute of Light Industry, Jinan250353, P.R. China. \\ ${ }^{\mathrm{c}}$ Department of Physics, Redeemers University Nigeria, Ogun State, Nigeria. \\ ${ }^{\mathrm{d}}$ Nonlinear Biomedical Physics, Department of Physics, Lancaster University, \\ Lancaster LA1 4YB, United Kingdom. \\ babaidowu@yahoo.com (B. A. Idowu), rongwei guo@163.com \\ (RongweiGuo), u.vincentelancaster.ac.uk (U. E. Vincent).
}

\begin{abstract}
Recently, we introduced a simple adaptive control technique for the synchronizationand stabilization of chaotic systems based on the Lasalle invariance principle. The method is very robust to the effect of noise and can use single-variable feedback toachieve the control goals. In this paper, we extend our studies on this technique tothe nonlinear gyroscopes with multi-system parameters. We show that our proposedadaptive control can stabilize the chaotic orbit of the gyroscope to its stable equilibrium and also realized the synchronization between two identical gyros even whenthe parameters are assumed to be uncertain. The designed controller is very simple relative to the system being controlled, employs only a single-variable feedbackwhen the parameters are known; and the convergence speed is very fast in all cases. We give numerical simulation results to verify the effectiveness of the technique andits robustness in the presence of noise.
\end{abstract}

\section{Key words:}

chaos synchronization, adaptive control, Nonlinear gyroscopePACS: 05.45.-a, 05.45.Pq, 05.45.Ac

\section{Introduction}

Chaotic dynamics has been studied and developed with much interest since the work of Lorenz in 1963 [1,2] which has led to the observation of chaotic behaviour in many systems. A chaotic system generally has complex dynamical behaviour arising from the unpredictability of the longterm future behaviour and irregularity.

Two prominent and leading applications in the development of chaos theory are chaos suppression or control and chaos synchronization. The emergence of these two areas in the study of nonlinear systems is traceable to the pioneering classical chaos control theory by Ott, Grebogi and Yorke[3] and the seminal work by Pecora and Carroll [4] respectively. Indeed, there are DOI : $10.5121 /$ ijccms.2013.2204 
practical situations where it is desirable to control chaotic behaviour so as to improve the performance of a dynamical system, eliminate undesirable behaviour of power electronics [5-7], avoid erratic fibrillations of heart beating, and so on. As a result, different techniques and methods have been proposed to achieve chaos control [8- 13] - which is stabilizing a desired unstable periodic solution or one of the systems equilibrium points. On the other hand, for synchronization, two systems are required to co-operate with each other, of which many potential applications abounds in secure communication systems, laser, biological systems and other areas $[10,11,14,15]$. For this reason, the study of chaos synchronization has grown and a wide variety of approaches have been proposed for the synchronization of chaotic systems [4,9-11,16-21].

Gyroscopes, from a purely scientific viewpoint show strange and interesting properties, and from engineering viewpoint, they have great utility in the navigation of rockets, aircrafts, spacecrafts and in the control of complex mechanical system. In the past years, the gyroscope have been found with rich phenomenon [22 - 24 ] for example, the symmetric gyroscope, when subjected to harmonic vertical base excitations, exhibit a variety of interesting dynamic behaviours that span the range from regular to chaotic motions $[23,24]$. The gyro is one of the most interesting and everlasting nonlinear dynamical systems, which displays very rich and complex dynamics, such as sub-harmonic and chaotic behaviours.

Several attempts have been made to control and synchronize the gyroscope system. The delayed feedback control, addition of periodic force and adaptive control algorithm have been utilized to control chaos in a symmetric gyro with linear-plus-cubic damping [24] and in Ref. [25] we used a technique that is based on backstepping approach that interlace the appropriate choice of Lyapunov function.Notwithstanding the success recorded by these methods, some drawbacks associated with their applications have been identified.

On the other hand, the synchronization of the symmetric gyroscope model presented in Ref [24] has been achieved using different methods. In Ref. [24] for instance, synchronization was achieved using four different kinds of one way coupling. The stability of the synchronization is subject to the verification that the conditional Lyapunov exponents of the subsystem is negative. This condition has however been proved not to be a sufficient condition for chaos synchronization due to some unstable invariant sets in the stable synchronization manifold lcite $\{$ manifold $\}$. However, whether or not this condition is a necessary or/and sufficient condition remains unresolved (see Refs. [27] and references therein).

In Ref. [28] the active control method was utilized. It is important to note that in practice it is difficult to find appropriate or threshold values for the feedback gains in the approaches used in Ref. [24] and this is also a topical issue and also the analysis in Ref.[24] were based on numerical simulations only. Similarly, the experimental design of nonlinear control inputs such as proposed in [25,28-32] are very difficult, due to the complexity of the control functions, especially when the system parameters are unknown due to inevitable perturbation by external inartificial factors. Although in [30-34] the sliding mode control was proposed that could be applicable to the above situations in the presence of parameter uncertainty [31]; however there were many assumptions to be made in the construction of the controllers. For example, in [30] the exact values of the functions are unknown due to parameter uncertainty; some upper bounds of uncertainties are necessary and also assumed that all the state variables of the master and slave systems are available for control design. 
International Journal of Chaos, Control, Modelling and Simulation (IJCCMS) Vol.2, No.2, June 2013

Whereas in [31] where the adaptive sliding mode (ASMC) is used for synchronizing the state trajectories of two chaotic gyros with unknown parameters and external disturbance, a switching surface is first proposed, thereafter an ASMC is derived, thus, if the switching surface is in error, the ASMC derived will be ineffective. Also, in this method, the controller demonstrates a discontinuous control law and the phenomenon of chattering will appear - this undesirable and has to be eliminated. In [32] the sliding mode controller was extended to achieve generalized projective chaos synchronization (GPS) for the gyroscope system subjected to dead-zone nonlinear inputs, where the drive-response system synchronized up to a constant scaling factor. Similar to other sliding mode approaches, two suitable sliding surfaces were proposed to ensure the stability of surfaces even when the control inputs contain dead-zone nonlinearity. The method also allows to arbitrarily direct the scaling factor onto a desired value. Similarly, it was reported that synchronization and anti-synchronization may co-exist in projective chaos synchronization of a dissipative gyroscope excited by a harmonic force with control input nonlinearity [35].

The synchronization for above situations is complex and not straightforward due to the problems associated with sliding surface design. More importantly, the design of simple controllers is very relevant for both theoretical research and practical applications, which we intend to achieve in our proposed method.

Recently, in ref [36] they studied the chaos suppression of the chaotic gyros in a given finite time, where they considered the effects of model uncertainties, external disturbances and fully unknown parameters. They designed a robust adaptive finite-time controller to suppress the chaotic vibration of the uncertain gyro. In similar manner, in [37], the problem of the finite-time synchronization of two uncertain chaotic gyros is discussed.

In year 2000, new experimental results demonstrate that chaos control can be accomplished using controllers that are very simple relative to the system being controlled [38]. For this reason, theoretical studies of chaos control and synchronization with simple adaptive control emerged [39-49]. In [45] a novel adaptive controller for achieving chaos and hyperchaos synchronization was proposed. This adaptive control method was used to realize the synchronization of coupled RCL-shunted Josephson junctions [50] the reduced-order synchronization of time-varying systems [51] and extended to realize the stabilization of the unified chaotic system [52].This adaptive control method has better advantages than the linear feedback method, since the feedback gain $\mathrm{k}_{1}$ is automatically adapted to a suitable gain $\mathrm{k}_{0}$ depending on the initial values. It is not only simple in comparison with other previous methods, but also suitable for all chaotic systems and hyperchaotic systems. In most cases, the controller can include only one feedback gain $k_{1}$ and the convergence speed is very fast.

In this paper, we first apply the method for stabilizing the chaotic orbits to the equilibrium point of the system. Secondly, we used our adaptive control method to synchronize two identical nonlinear gyroscopes and thirdly we extend the method to achieve synchronization in the gyros with unknown parameters, which configures a real-life situation. Fourthly, as a further advancement, the robustness of the method is verified by adding noise, and the synchronization in this situation was achieved. The designed simple controller ensures stable controlled and synchronized states for the nonlinear gyros. Finally, numerical simulations are implemented to verify the results. 


\section{Theory}

\subsection{Adaptive control for chaos synchronization}

In this section, we introduce the adaptive control method [45] briefly. Let a chaotic (master) system be given as,

$\dot{x}=f(x)$

Where $\quad x=\left(x_{1}, x_{2}, \cdots, x_{n}\right)^{T} \in R^{n}, \quad f(x)=\left(f_{1}(x), f_{2}(x), \cdots, f_{n}(x)\right)^{T}: R^{n} \rightarrow R^{n} \quad$ is a nonlinear vector function. Without loss of generality, let $\Omega \subset R^{n}$ be a chaotic bounded set of (1) which is globally attractive. For the vector function $f(x)$ we give a general assumption.

Assumption 1. $\forall_{X}=\left(x_{1}, x_{2}, \cdots, x_{n}\right)^{T} \in \Omega$ and $y=\left(y_{1}, y_{2} \cdots, y_{n}\right)^{T} \in \Omega$ there exists a constant $l>0$ satisfying

$\left|f_{i}(x)-f_{i}(y)\right| \leq l|x-y|_{\infty}, i=1,2, \cdots, n$

where $|\mathrm{x}-\mathrm{y}||x-y|_{\infty}$ is the $\infty$-norm of $x-y . i . e .,|x-y|_{\infty}=\underset{j}{\max }\left|x_{j}-y_{j}\right|, j=1,2, \cdots, n$.

Remark 1. This condition is very loose, and in fact, holds as long as $\partial f_{i} / \partial x_{j}(i, j=1,2, \cdots, n)$ are bounded. Thus, the class of systems in the form of (1) and (2) include almost all well-known finite-dimensional chaotic and hyperchaotic systems.

The corresponding slave system to system (1) is as follows,

$\dot{y}=f(y)+k_{1}(y-x)$

where the controller $u=k_{1}=\left(k_{1} e_{1}, k_{1} e_{2}, \cdots, k_{1} e_{n}\right)^{T}, e_{i}=y_{i}-x_{i}$. Unlike the usual linear feedback control, the feedback gain $k_{1}$ is duly adapted according to the following update law,

$\dot{k_{1}}=-\gamma \sum_{i=1}^{n} e_{i}^{2}$

Where $\gamma$ is an arbitrary positive constant. The controller $u=k_{1} e$ can realize the synchronization of the master and slave chaotic systems (1) and (2).

Remark 2. The feedback gain $k_{1}$ is automatically adapted to a suitable strength $k_{0}$ depending on the initial values, which is significantly different from the well known linear feedback.

Remark 3. The controller $u=k_{1} e$ can employ only one feedback term $e_{i}$ for some chaotic systems, the feedback term $e_{i}$ is selected by the condition: if $e_{i}=0$ then $e_{j}=0, j=$ $1,2, \cdots n, j \neq i$, so that the set $E=\left\{\left(e, k_{1}\right) \in R^{n+1} \mid e=0, k_{1}=k_{0}\right\}$ and the above conclusion is obtained.

\subsection{Adaptive control for chaos synchronization with unknown parameters}

In our previous paper [51], we obtained a novel adaptive controller for chaos synchronization with unknown parameters. This is introduced in brief herein. Consider a nonlinear dynamical system

$\dot{x}=f(x)+g(x) p$, 
International Journal of Chaos, Control, Modelling and Simulation (IJCCMS) Vol.2, No.2, June 2013

Where $x=\left(x_{1}, x_{2}, \cdots, x_{n}\right)^{T} \in \mathrm{R}^{n}$ denotes the state variables, $p=\left(p_{1}, p_{2}, \cdots, p_{k}\right)^{T} \in \mathrm{R}^{k}$ denotes the uncertain parameters, $f(x)=\left(f_{1}(x), f_{2}(x), \cdots, f_{n}(x)\right)^{T}$ and $g(x)=[g(x)]_{n x k}$ represent differential nonlinear vector function and matrix function respectively. The vector function $f(x)$ meets the Assumption 1.

We consider model (5) as the master system and introduce a controlled slave system

$\dot{y}=f(y)+g(y) p+u$,

Where $y=\left(y_{1}, y_{2}, \cdots, y_{n},\right)^{T} \in \mathrm{R}^{n}$ denotes the state variables, and $u=\left(u_{1}, u_{2}, \cdots, u_{n}\right)^{T}$ is a controller. The main goal is to design a suitable controller $u$ to synchronize the two identical systems in spite of their uncertain parameters. We denote the synchronization error between the two systems as $e=y-x \in \mathrm{R}^{n}$ and subtract system (5) from system (6) and thus obtain the error dynamical system

$\dot{e}=f(y)-f(x)+[g(y)-g(x)] p+u$,

We can introduce the control function

$u=-[g(y)-g(x)] \hat{P}+k_{1} e$,

Where $\hat{p}$ is the estimate of $p$, and $k_{1} e=\left(k_{1} e_{1}, k_{1} e_{2}, \cdots, k_{1} e_{n}\right)^{T} \in \mathrm{R}^{n}$ is the linear feedback control with the updated gain $k_{1} \in \mathrm{R}^{1}$ Thus, the synchronization error system is reduced to

$\dot{e}=[f(y)-f(x)]+[g(y)-g(x)] \tilde{p}+k_{1} e$,

Where $\tilde{p}=p-\hat{p}$ is the parameter estimation mismatch between the real value of the unknown parameter and its corresponding estimated value. Then the above discussion can be summarized in the following theorem.

Theorem 1 If the estimations of the unknown parameters and the feedback gain contained in the adaptive controller (8) are updated by the following laws

$\left\{\begin{array}{c}\dot{\hat{p}}=[g(y)-g(x))^{T} e, \\ \dot{k}_{1}=-\gamma e^{T} e=-\gamma \sum_{l=1}^{n} e_{l}^{2},\end{array}\right.$

then, the synchronization between system (5) and (6) will be achieved.

Remark 4. The control term $k_{1} e$ can include only one feedback term $e_{i}$ for some chaotic systems. The feedback term $e_{i}$ is selected based on the condition: if $e_{i}=0$ then $e_{j}=0, j=$ $1,2, \cdots n, j \neq i$, therefore the set $E=\left\{e, \hat{p}, k_{1}\right) \in \mathrm{R}^{n+k+1} \mid e=0, \hat{p}=0, k_{1}=-k^{*}$ so that the conclusion in (10) is obtained.

\subsection{Adaptive control for stabilization of chaotic system}

Here, we extend our adaptive control method [52] to stabilize a chaotic orbit. Given a chaotic system (1), for the vector function $f(x)$, we give a general assumption which is similar to the assumption 1. 
International Journal of Chaos, Control, Modelling and Simulation (IJCCMS) Vol.2, No.2, June 2013

Assumption 2. $\forall x=\left(x_{1}, x_{2}, \cdots, x_{n}\right)^{T} \in \Omega$, there exists a constant $l>0$ satisfying

$\left|f_{i}(x)\right| \leq l|x|_{\infty}, i=1,2, \cdots n$

Where $|x|_{\infty}$ is the $\infty$-norm of $x$, i.e., $|x|_{\infty}=\max _{j}\left|x_{j}\right|, j=1,2, \cdots, n$

Remark 5. This condition is easily met, and in fact, holds as long as $\frac{\partial f_{i}}{\partial x_{j}}(i, j=1,2, \cdots, n)$ are bounded. Therefore the class of systems in the form of (1) and (11) include almost all well-known finite-dimensional chaotic and hyper-chaotic systems.

In order to stabilize the chaotic orbits in (1) to its equilibrium point $x^{*}=0$ we introduce the adaptive feedback controller to system (1):

$\dot{x}=f(x)+u=f(x)+k_{1}(x-0)=f(x)+u$

where the controller $u=k_{1} x=\left(k_{1} x_{1}, k_{1} x_{2}, \cdots, k_{1} x_{n}\right)^{T}$. The feedback gain $k_{1}$ is adapted according to the following update law,

$\dot{k}_{1}=-\gamma \sum_{i=1}^{n}\left(x_{i}-0\right)^{2}=-\gamma \sum_{i=1}^{n} x_{i}^{2}$

Where $\gamma$ is a positive constant. System (12) and (13) are assumed to be the augment system and by introducing a positive definite Lyapunov function,

$V=\frac{1}{2} \sum_{i=1}^{n} x_{i}^{2}+\frac{1}{2} \frac{1}{\gamma}\left(k_{1}+L\right)^{2}$,

Where $L$ is a sufficiently large positive constant, i.e., $L \geq n l$. Then, we give the following result.

Theorem 2. Starting from any initial values of the augment system, the orbits $\left(x(t), k_{1}(t)^{T}\right.$ converge to $\left(x^{*}, k_{0}\right)^{T}$ as $t \rightarrow \infty$, where, $k_{0}$ is a negative constant depending on the initial value. That is, the adaptive feedback controller stabilizes the chaotic orbits to its equilibrium point $x^{*}$.

Proof. By differentiating the Lyapunov function $V$ along the trajectories of the augment system, we obtain

$$
\begin{gathered}
\dot{V}=\sum_{i=1}^{n} x_{i} \dot{x}_{l}+\frac{1}{\gamma}\left(k_{1}+L\right) \dot{k}_{1} \\
=\sum_{i=1}^{n} x_{i}\left(f_{i}(x)+k_{i} x_{i}\right)-\left(k_{1}+L\right) \sum_{i=1}^{n} x_{i}^{2} \\
=\sum_{i=1}^{n} x_{i} f_{i}(x)-L \sum_{i=1}^{n} x_{i}^{2}
\end{gathered}
$$

Obviously, $\dot{V}=0$ if and only if $x_{i}=0, i=1,2, \cdots, n$,then the set

$E=\left\{\left(x, k_{1}\right) \in R^{n+1} \mid \dot{V}(x)=0\right.$ is the largest invariant set for the augment system. According to the well known LaSalle invariance principle, the Theorem 1 is obtained. 
International Journal of Chaos, Control, Modelling and Simulation (IJCCMS) Vol.2, No.2, June 2013

Remark 6. If $x^{*} \neq 0$ is an equilibrium point of the chaotic system, this method is also easily applicable by means of a coordinate transformation.

Remark 7. The feedback gain $\mathrm{k}_{1}$ is also automatically adapted to a suitable strength $k_{0}$ depending on the initial values.

Remark 8. The controller $u=k_{1} x=\left(k_{1} x_{1}, k_{1} x_{2}, \cdots, k_{1} x_{n}\right)^{T}$ can include only a single-variable feedback term $x_{i}$, which is selected based on the condition: if $x_{i}=0$ then $x_{j}=0, j=$ $1,2 \cdots n, j \neq i$, therefore the set $E=\left\{\left(x, k_{1}\right) \in R^{n+1}\left|x=0, k_{1}=k_{1}\right|\right.$ and the above conclusion is reached.

\section{Applications to gyroscopes}

The model system which we study is the gyroscope which has attributes of great utility to navigational, aeronautical and space engineering [24], and have been widely studied. Generally, gyros are understood to be devices which rely on inertial measurement to determine changes in the orientation of an object. Gyros are recently finding application in automotive systems for Smart Braking System, in which different brake forces are applied to the rear tyres to correct for skids, for sensing angular motion in airplane automatic pilots, rocket-vehicle launch guidance, etc. Recently, Chen, presented the dynamic behaviour of a symmetric gyro with linear-plus-cubic damping, and subjected to a harmonic excitation. Based on Lyapunov analysis, sufficient conditions for the stability of the equilibrium points of the system were derived.

The equation governing the motion of the symmetric gyro with linear-plus-cubic damping is governed by the following equation in term of the angle $\theta$ [24]:

$$
\ddot{\theta}+\alpha^{2} \frac{(1-\cos \theta)^{2}}{\sin ^{3} \theta}-\beta \sin \theta+c_{1} \dot{\theta}+c_{2} \dot{\theta}^{3}=(f \sin \omega t) \sin \theta
$$

Where $f \sin \omega t \quad$ is a parametric excitation, $c_{1} \dot{\theta}$ and $c_{2} \dot{\theta}^{3}$ are linear and nonlinear damping, respectively and $\alpha^{2} \frac{(1-\cos \dot{\theta})^{2}}{\sin ^{3} \theta}-\beta \sin \theta$ is a nonlinear resilence force. After necessary transformation, the gyroscope equation in non-dimensional form can be written as

$\dot{x_{1}}=x_{2}$

$$
\dot{x_{2}}=g\left(x_{1}\right)-a x_{2}-b x_{2}^{3}+\beta \sin x_{1}+(f \sin \omega t) \sin x_{1}
$$

where the function

$g\left(x_{1}\right)=-\alpha^{2} \frac{\left(1-\cos x_{1}\right)^{2}}{\sin ^{3} x_{1}}$

The gyro undergoes the period-doubling bifurcation leading to chaotic behaviour when the parameter $f$ is used as the bifurcation parameter. In particular, a chaotic attractor is observed for the following system parameters: $\alpha^{2}=100, \beta=1, a=0.5, \omega=2, f=35.3$. Figure 1 show the phase trajectory for these parameters with initial conditions of $\left(x_{1}, x_{2}\right)=(1,-1)$. 
International Journal of Chaos, Control, Modelling and Simulation (IJCCMS) Vol.2, No.2, June 2013

\subsection{Stabilization of the nonlinear gyroscope}

According to section 2.3 , let $V\left(x, k_{1}\right)=\frac{1}{2}\left(x_{1}^{2}+x_{2}^{2}+\left(k_{1}+L\right)^{2}\right)$, then $\dot{V}(x)=0$, i.e.

$x_{1} x_{2}+x_{2} g\left(x_{1}\right)+G G_{*}=L \sum_{i=1}^{2} x_{i}^{2}+a x_{2}^{2}+b x_{2}^{4}$,

Where $G G_{*}=\beta x_{2} \sin x_{1}+\left(f x_{2} \sin \omega t\right) \sin x_{1}$. Obviously, if $x_{1}=0$ (the left hand side of the above equation), then $x_{2}=0$ (according to the right hand side of the above equation), thus $E=\left\{\left(x_{1}, x_{2}, k_{1}\right) \in R^{3} \mid \dot{V}(x)=0\right\}=\left\{\left.\left(x_{1}, x_{2}, k_{1}\right) \in R^{3}\right|_{x=0, k_{1}=k_{0}}\right.$

So, we can select the controller $u=k_{1} x=\left(k_{1} x_{1}, 0\right)^{T}$ and set $\dot{k_{1}}=-x_{1}^{2}$ (selecting $\gamma=$ 1 ). Then the controlled system is as follows,

$\dot{x_{1}}=x_{2}+k_{1} x_{1}$,

$\dot{x_{2}}=g\left(x_{1}\right)-a x_{2}-b x_{2}^{3}+\beta \sin x_{1}+f \sin \omega t \sin x_{1}$

Therefore the gyroscope system is stabilized to its equilibrium state with the controller $u=$ $k_{1} x=\left(k_{1} x_{1}, 0\right)^{T}$.

In what follows, we give numerical verification of the above theoretical results. We select the values of the initial states of the chaotic system (16) as $x_{1}(0)=1, x_{2}(0)=2$ with the initial value of the controller $k_{1}(0)=-1$ and selecting $\gamma=1$. Figure 2 shows that the gyros system stabilized to the zero solution while Figure 3 shows how the feedback gain $k_{1}$ tends to a negative constant as $t \rightarrow \infty$.

\subsection{Synchronization of the nonlinear gyro with known system parameters}

The corresponding slave system to (16) is as follows

$\dot{y}_{1}=y_{2}+u_{1}$

$$
\dot{y}_{2}=g\left(y_{1}\right)-a y_{2}-b y_{2}^{3}+\beta \sin y_{1}+(f \sin w t) \sin y_{1}+u_{2}
$$

where the controller $u=\left(u_{1}, u_{2}\right)^{T}$ is to be determined. According to section 2.1, let

$V\left(e, k_{1}\right)=\frac{1}{2}\left(e_{1}^{2}+e_{2}^{2}+\left(k_{1}+L\right)^{2}\right)$, then $\dot{V}(e)=0$, i.e.

$e_{1} e_{2}+e_{2}\left(g\left(y_{1}\right)-g\left(x_{1}\right)\right)+G_{x x}=G_{y y}+L\left(e_{1}^{2}+e_{2}^{2}\right)$

Where $G_{x x}=2 \sin \left(\frac{e_{1}}{2}\right) \cos \left(\frac{y_{1}+x_{1}}{2}\right)(\beta+f \sin w t) e_{2}^{2}$ and $G_{y y}=a e_{2}^{2}+b e_{2}^{2}\left(y_{1}^{2}+y_{1} x_{1}+x_{1}^{2}\right)$.

Obviously, if $e_{1}=0$ (from the left hand side of eq. (21)), then $e_{2}=0$ (according to the right hand side of eq. (21)). Thus; $E=\left(e_{1}, e_{2}, k_{1}\right) \subset R^{3}\left|\dot{V}(e)=0=\left(e_{1}, e_{2}, k_{1}\right) \subset R^{3}\right| e=0, k_{1}=k_{0}$. So, we can select the controller $u=k e=\left(k_{1} e_{1}, 0\right)^{T}$ and set $\dot{k}_{1}=-e_{1}^{2}$ (selecting $\left.\gamma=1\right)$. Therefore the chaos synchronization between the system (16) and (20) is realized. 
International Journal of Chaos, Control, Modelling and Simulation (IJCCMS) Vol.2, No.2, June 2013

To numerically verify the above, we select the initial state values of the master system (16) as $x_{1}(0)=1, x_{2}(0)=2$ and that of the slave system (20) as $y_{1}(0)=-1, y_{2}(0)=2$, with the initial value of the controller $k_{1}(0)=-1$; and setting $\gamma=1.0 \mathrm{n}$ application of the controller, Figure 4 shows that the error system is asymptotically stable to zero while Figure 5 shows how the feedback gain $k_{1}$ tends to a negative constant as $t$ tends to $\infty$.

\subsection{Synchronization of the nonlinear gyro with unknown system parameters}

If the parameters $a, b$, and $\beta$ of the master system (16) are unknown, we can still realize the full synchronization. According to section 2.2, the corresponding slave system is as follows

$$
\begin{aligned}
& \dot{y_{1}}=y_{2}+u_{1}, \\
& \dot{y_{2}}=g\left(y_{1}\right)-a y_{2}-b y_{2}^{3}+\beta \sin y_{1}+f \sin \omega t y_{1}+u_{2},
\end{aligned}
$$

where the parameters $a, b, \beta$ are unknown and the controller $u=\left(u_{1}, u_{2}\right)^{T}$ is to be determined.

According to the general framework given in Section 2.2 and the remark 4, the adaptive controller is designed as follows:

$$
\begin{aligned}
& u_{1}=k_{1} e_{1} \\
& u_{2}=\left(y_{2}-x_{2}\right) \hat{a}+\left(y_{2}^{3}-x_{2}^{3}\right) \hat{b}-\left(\sin y_{1}-\sin x_{1}\right) \hat{\beta}
\end{aligned}
$$

The updated laws for the parameter estimations $\hat{a}, \hat{b}, \hat{\beta}$ and the feedback gain $k_{1}$ in the above controller are given by

$$
\left\{\begin{array}{c}
\dot{\hat{a}}=\left(y_{2}-x_{2}\right) e_{2}, \\
\dot{\hat{b}}=\left(y_{2}^{3}-x_{2}^{3}\right) e_{2}, \\
\dot{\hat{\beta}}=\left(\sin y_{1}-\sin x_{1}\right) e_{2} \\
\dot{k_{1}}=-\gamma e_{1}^{2}
\end{array}\right.
$$

With (23) and (24), chaos synchronization between the system (16) and (22) is realized.

We verify the above numerically by selecting the initial states values of the master system (16) as $x_{1}(0)=1, x_{2}(0)=2$ and that of the slave system $(21)$ as $y_{1}(0)=-1, y_{2}(0)=2$. The parameter estimations $(\hat{a}, \hat{b}, \hat{\beta})=(0.4,0.01,0.5)$ and feedback gain $k_{1}(0)=-1$. For $\gamma=1$, Figure 6 shows that the error system approaches zero asymptotically as $t \rightarrow \infty$;while Figure 7 shows how the feedback gain $k_{1}$ tends to a negative constant as $t \rightarrow \infty$.

Finally, we verify the robustness of this method by adding noise to the slave system (20), which then becomes,

$$
\begin{aligned}
& \dot{y}_{2}=y_{2}+k_{1} e_{1}+\text { rand }, \\
& \dot{y}_{2}=g\left(y_{1}\right)-a y_{2}-b y_{2}^{3}+\beta \sin y_{1}+f \sin \omega t \sin y_{1},
\end{aligned}
$$


International Journal of Chaos, Control, Modelling and Simulation (IJCCMS) Vol.2, No.2, June 2013

whererand is a stochastic random number uniformly distributed in the interval $(0,1)$.

Using the same initial values and parameters as in Figure 2, Figure 8 shows the asymptotic convergence to zero of the error system as $t \rightarrow \infty$ in the controlled state and the feedback gain $k_{1}$ tends to a negative constant as $t \rightarrow \infty$; implying that the gyro system are synchronized despite the presence of noise. Also, in Figure 9 and Figure 10 the error system is also asymptotically stable to zero and the feedback gain $k_{1}$ tends to a negative constant as $t \rightarrow \infty$ when noise is added to the slave system for the gyroscope systems with unknown parameters, respectively.

\section{Conclusion}

In conclusion, we investigated the control and synchronization of chaos in nonlinear gyros. Firstly, we obtained a simple adaptive control law for stabilizing chaotic orbits of the gyros to its equilibrium point. Secondly, we extend our adaptive control method to synchronize a driveresponse system of nonlinear gyros, with known and unknown parameters. The designed simple controller ensures stable controlled and synchronized states for two identical nonlinear gyros. In addition, the synchronization is efficient in the presence of noise for the three cases mentioned above. The controller designed is very simple relative to the system being controlled, it includes only one feedback gain and the convergence speed is very fast; and we have employed numerical simulations to verify the results.

\section{Acknowledgements}

U E Vincent has been supported by The British Academy, The Royal Academy of Engineering and The Royal Society of London, through the Newton International Fellowships.

\section{References}

[1] E. N. Lorenz, Deterministic non-periodic flows. Journal of Atmospheric Science20 (1963) 130-141.

[2] C. Sparrow, The Lorenz equations: Bifurcations, chaos and strange attractor.Springer-Verlag New York 1982.

[3] E. Ott, C. Grebogi, J. A. Yorke, Controlling Chaos. Physics Review Letters 64(1990) 1196-1199.

[4] L. M. Pecora , TL Carroll, Synchronization in chaotic systems. Physics ReviewLetters 64 (1990) $821-$ 824.

[5] H. O. Wang, E. H. Abed, Control of nonlinear phenomena at the inceptionof voltage collapse. In Proc. 1993 American control conference, San FranciscoJune, (1993) 2071 -2075.

[6] E. H. Abed, P. P. Varaiya, Nonlinear oscillations in power systems. InternationalJournal of Electric Power and Energy System 6 (1989) 37-43.

[7] E. H. Abed, J.-H. Fu (1986), Local Feedback stabilization and bifurcationcontrol, I. Hopf bifurcation.Systems and Control Letters 7,11-17.

[8] T. Kapitaniak, Controlling Chaos - Theoretical \& Practical Methods in Non-linear Dynamics. Academic Press, London, 1996.

[9] G. Chen and X. Dong, From Chaos to Order: Methodologies, Perspectives andApplications. Singapore, World Scientific, 1998.

[10] A. S. Pikovsky, M. G. Rosenblum, J. Kurths, Synchronization - A UnifiedApproach to Nonlinear Science, Cambridge University Press, Cambridge, 2001.

[11] M. Lakshmanan and K. Murali, Chaos in Nonlinear Oscillators: Controlling andSynchronization, World Scientific, Singapore, 1996. 
International Journal of Chaos, Control, Modelling and Simulation (IJCCMS) Vol.2, No.2, June 2013

[12] A. L. Fradkov, A. Yu. Pogromsky, Introduction to Control of Oscillations andChaos World Scientific, Singapore, 1996.

[13] H.-T. Yau and C. L. Chen, Controlling Lorenz Chaotic System using AdaptiveController with Input Saturation, Chaos, Solitons\& Fractals, 34 (2007)1567-1774.

[14] A. Stefanski, T. Kapitaniak, Synchronization of two chaotic oscillators via anegative feedback mechanism, Chaos Solitons \& Fractals 40 (2003) 5175-5185.

[15] T. Kapitaniak, Continuous control and synchronization in chaotic systems, Chaos, Solitons\& Fractals 6 (1995) 237-244.

[16] H.-T. Yau and C. S. Shieh, Chaos synchronization using fuzzy logic controller,Nonlinear Analysis B: Real World Applications, 9 (2008) 1800-1810.

[17] H.-T. Yau and J. J. Yan, Chaos synchronization of different chaotic systemsubjected to input nonlinearity, Applied Mathematics and Computation 197(2008) 775-788.

[18] A. R. Sahab and M. T. Ziabari, Anti-synchronization of two differenthyperchaotic systems via active generalized backstepping method, Int. J ofChaos, Control, Modelling and Simulation, 2(1) (March 2013), 1-9.

[19] S. Vaidyanathan, Active Controller design for regulating the output of theSprott-P System, Int. J of Chaos, Control, Modelling and Simulation, 2(1)(March 2013), 11-20.

[20] Y.-L. Ling, Y. Sun and X. Dai, Robust Control for an uncertain LCL resonantICPTsystem using LMI method, Control Engineering Practice, 21 (2013), 31-41.

[21] Y. Xu, W. Zhou and J. Fang, Adaptive bidirectionally coupled synchronizationof chaotic systems with unknown parameters, Nonlinear Dyn66 (2011), 67-76.

[22] R. V. Dooren, Comments on 'Chaos and chaos synchronization of a symmetricgyro with linear-pluscubic damping', Journal of Sound and Vibration 268(2003) 632-634.

[23] Z. M. Ge, H. K. Chen, Bifurcations and chaos in a rate gyro with harmonicexcitation, Journal of Sound and Vibration 194 (1996) 107-117.

[24] H. K. Chen, Chaos and chaos synchronization of a symmetric gyro with linear-plus-cubic damping, Journal of Sound and Vibration 255 (2002) 719-740.

[25] B. A. Idowu, U. E. Vincent, A. N. Njah, Control and Synchronization of chaosin nonlinear gyros via backstepping design, International Journal of NonlinearScience 5 (2008) 11-19.

[26] J. F. Heagl, T. L. Carroll, and L. M. Pecora, Desynchronization by periodicorbits, Physical Review E 52 (1995) R1253 - R1256.

[27] J. W. Shuai, K. W. Wong, and L. M. Cheng, Synchronization of spatiotempoalchaos with positive conditional Lyapunov exponents, Physical Review E 56(1997) 2272-2275.

[28]. Lei, Wei Xu, HongchanZheng, Synchronization of two chaotic nonlineargyros using active control, Physics Letter A343 (2005) 153-158.

[29] H.-T. Yau, Nonlinear rule-based controller for chaos synchronization of twogyros with linear-pluscubic damping, Chaos Solitons\& Fractals 34 (2007) 1357-1365.

[30] H. Salarieh, A. Alasty, Chaos synchronization of nonlinear gyros in presence ofstochastic excitation via sliding mode control, Journal of Sound and Vibration313 (2008) 760-771.

[31] J.-J. Yan, M.-L.Hung, Teh-Lu Liao, Adaptive Sliding mode control forsynchronization of chaotic gyros with fully unknown parameters, Journal ofSound and Vibration 298 (2006) 298-306.

[32] H. -T. Yau, Generalized projective chaos synchronization of gyroscope systemssubjected to deadzone nonlinear inputs, Physics Letters A 372 (2008) 2380-2385.

[33] G. L. Shi and W. Shen, Hybrid control of a parallel platform based on pneumatic artificial muscles combining sliding mode controller and adaptive fuzzy CMAC,Control Engineering Practice21 (2013) 76-86.

[34] S. Vaidyanathan, Hybrid synchronization of hyperchaotic Liu systems viasliding mode control, Int. J of Chaos, Control, Modelling and Simulation, 1(1)(September 2012), 1-12.

[35] H. -T. Yau, Synchronization and anti-synchronization coexist in two-degree-of-freedom dissipative gyroscope with nonlinear inputs, Nonlinear Analysis: RealWorld Applications 9 (2008) 2253 - 2261.

[36] M. P. Aghababa and H. P. Aghababa, Chaos suppression of uncertain gyros in a given infinite time, Chin.Phys.B 21(11) (2012) 110505.

[37] M. P. Aghababa, A novel adaptive infinite-time controller for synchronizingchaotic gyros with nonlinear inputs, Chin. Phys. B 20(9) (2011) 090505. 
International Journal of Chaos, Control, Modelling and Simulation (IJCCMS) Vol.2, No.2, June 2013

[38] N. J. Corron, S.D. Pethel, B. A. Hopper, Controlling Chaos with simpleLimiters, Physical Review Letters 84 (2000) 3835-3838.

[39] D. Huang, Stabilizing Near-Nonhyperbolic Chaotic Systems with Applications,Physical Review Letters 93 (2004) 214101.

[40] D. Huang, Simple adaptive-feedback controller for identical chaossynchronization, Physical Review E 71 (2005) 037203.

[41] D. Huang, Adaptive-feedback control algorithm, Physical Review E 73 (2006)066204.

[42] D. Huang, Synchronization in adaptive weighted networks, Physical Review E74 (2006) 046208.

[43] M. Chen, D. Zhou, Synchronization in uncertain complex networks, Chaos 16(2006) 013101.

[44] J. Cao and J. Lu, Adaptive synchronization of neural networks with or without time-varying delay, Chaos 16 (2006) 013133.

[45] R. W. Guo, A simple adaptive controller for chaos and hyperchaossynchronization, Physics Letters A372 (2008) 5593-5597.

[46] X. Wang and Y. Wang, Adaptive control for synchronization of a four-dimensional chaotic system via a single variable, Nonlinear Dyn65 (2011) 311-316.

[47] Z. Sun, G. Si and F. Min, Adaptive modified function projective synchronization and parameter identification of uncertain hyperchaotic (chaotic) systems withidentical or non-identical structures, Nonlinear Dyn68 (2012) 471-486.

[48] M. Roopaei, M. Z. Jahromi and S. Jafari, Adaptive gain fuzzy sliding modecontrol for the synchronization of nonlinear chaotic gyros, Chaos 19 (2009)013125.

[49] Z. R. Xun and Y. Shi-Ping, A single adaptive controller with one variablefor synchronization of fractional-order chaotic systems, Chin. Phys. B 21(80)(2012) 080505.

[50] R. Guo, U.E. Vincent, B. A. Idowu, Synchronization of chaos in RCL-shuntedJosephson junction using a simple adaptive controller, PhysicaScripta79 (2009)035801-6.

[51] U.E. Vincent, RongweiGuo, A simple adaptive control for full andreduced-order synchronization of uncertain time-varying chaotic systems,Communication in Nonlinear Science and Numerical Simulation 14 (2009)3925-3932.

[52] R. Guo, U.E. Vincent, Control of a unified chaotic system via single variablefeedback, Chinese Physics Letters 26 (2009) 090506.

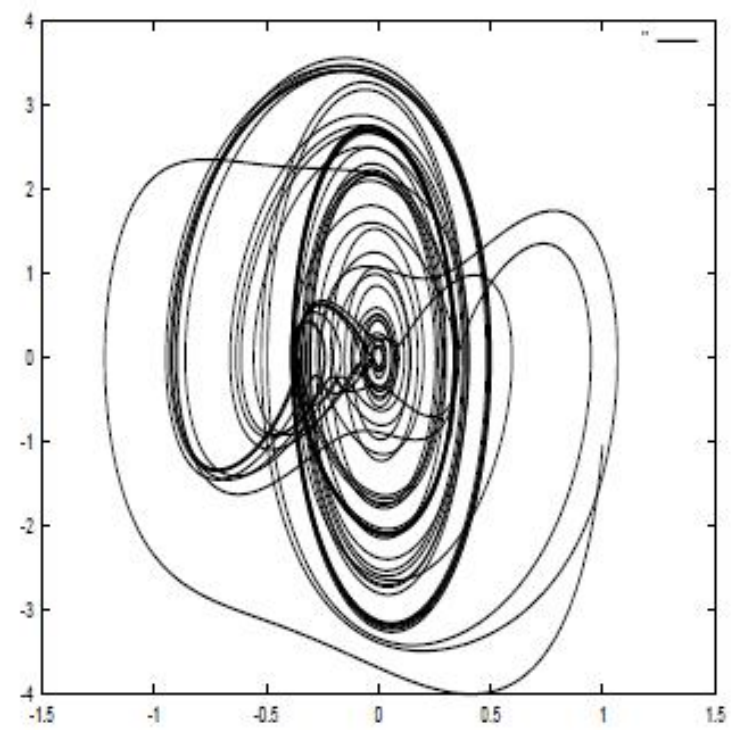

Fig. 1. The phase portrait of the chaotic gyro attractor described by Equation (16);initial values are $x_{1}=1, x_{2}=-1$. 
International Journal of Chaos, Control, Modelling and Simulation (IJCCMS) Vol.2, No.2, June 2013

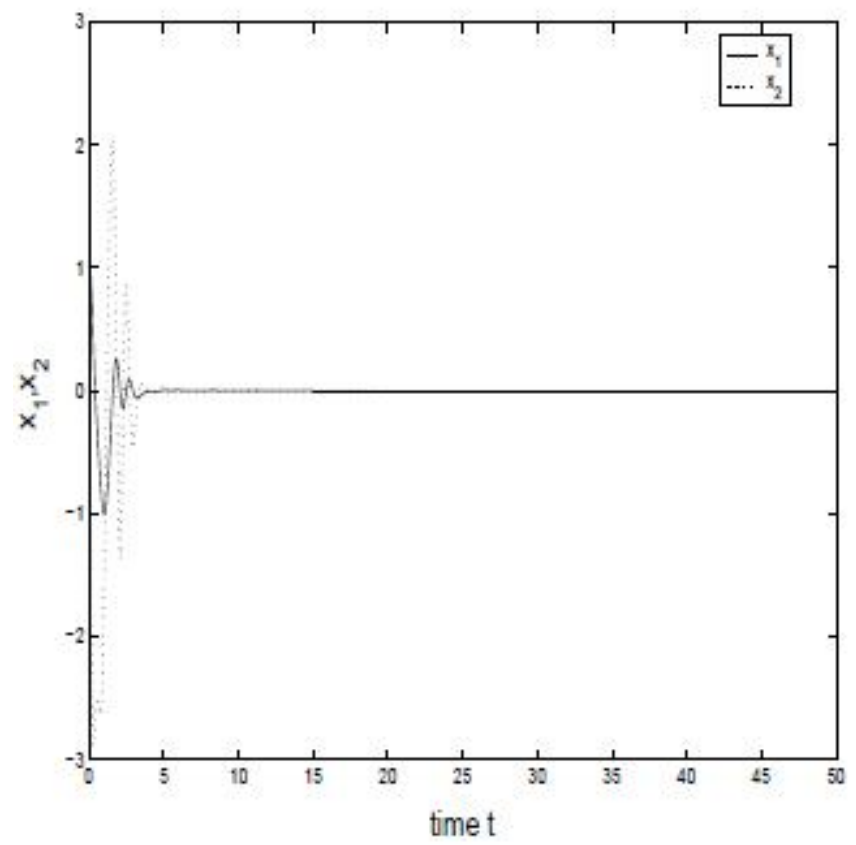

Fig. 2. The gyros system is asymptotically stable to zero as $t$ tends to $\infty$ in thecontrolled state, where the initial values are $x_{1}=1, x_{2}=2$ and the controller gain $k_{1}=-1$, selecting $\gamma=1$.

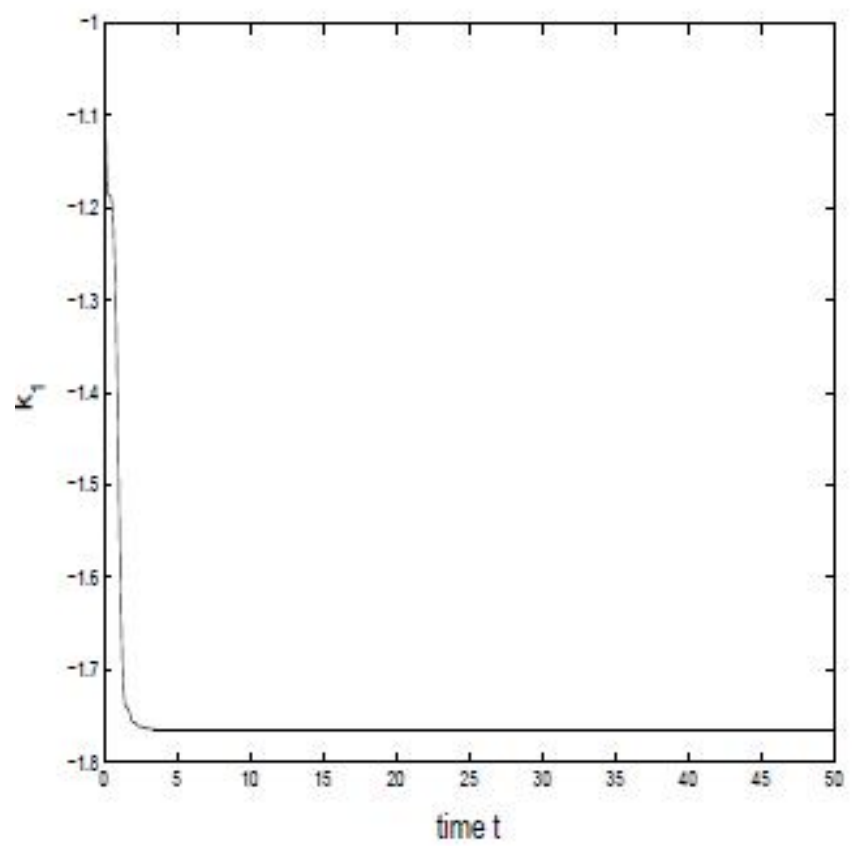

Fig. 3. The feedback gain $k_{1}$ tends to a negative constant ast $\rightarrow \infty$ in thecontrolled state and initial values as well as parameters are as in Figure 2. 
International Journal of Chaos, Control, Modelling and Simulation (IJCCMS) Vol.2, No.2, June 2013

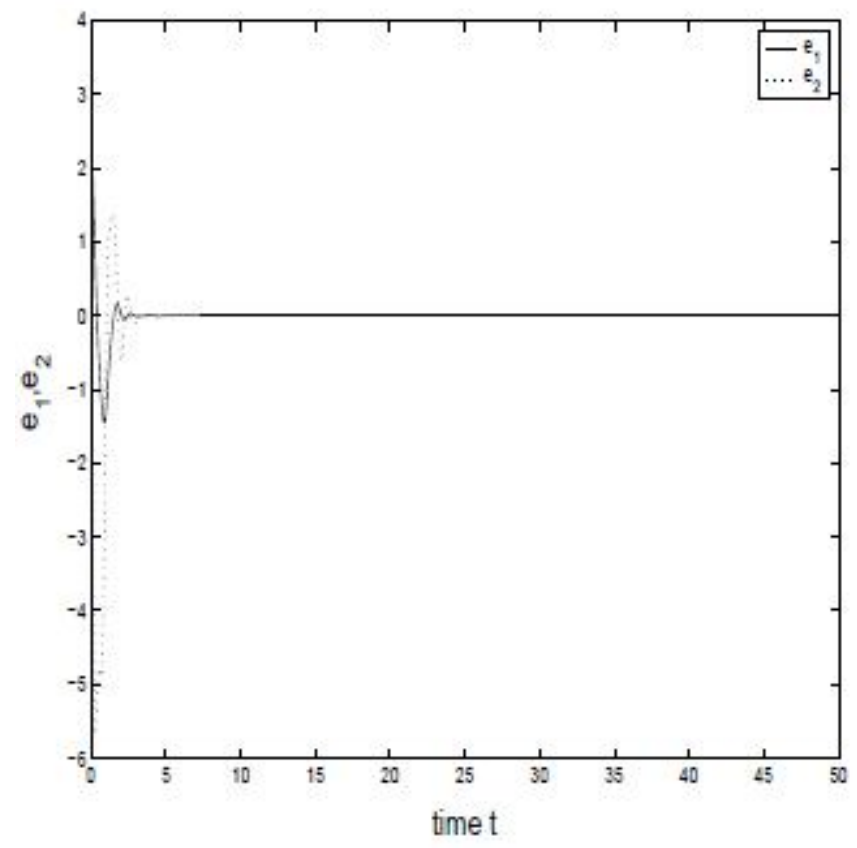

Fig. 4. The error system is asymptotically stable to zero as $\mathrm{t}$ tends to $\infty$ whensynchronization of identical gyros with different initial conditions is achieved for $x_{1}=1, x_{2}=2$ for the master system and $y_{1}=-1, y_{2}=2$ for the slave system.

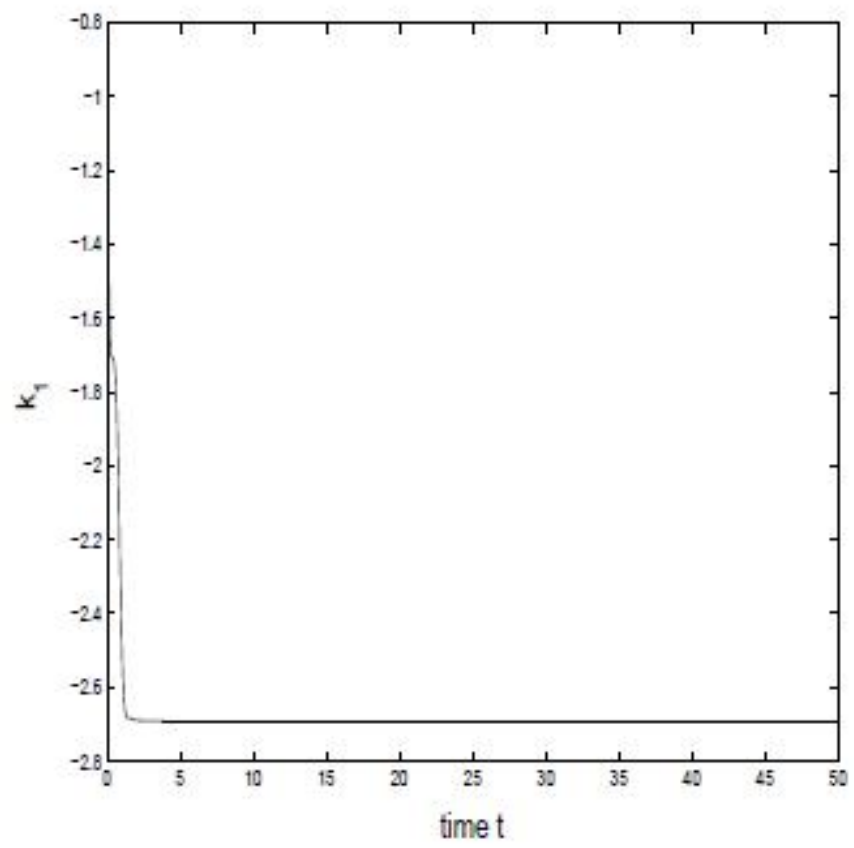

Fig. 5. The feedback gain $k_{1}$ tends to a negative constant as $\mathrm{t}$ tends to $\infty$ whensynchronization of identical gyros with different initial conditions is achieved andall initial values are as in Figure 4. 
International Journal of Chaos, Control, Modelling and Simulation (IJCCMS) Vol.2, No.2, June 2013

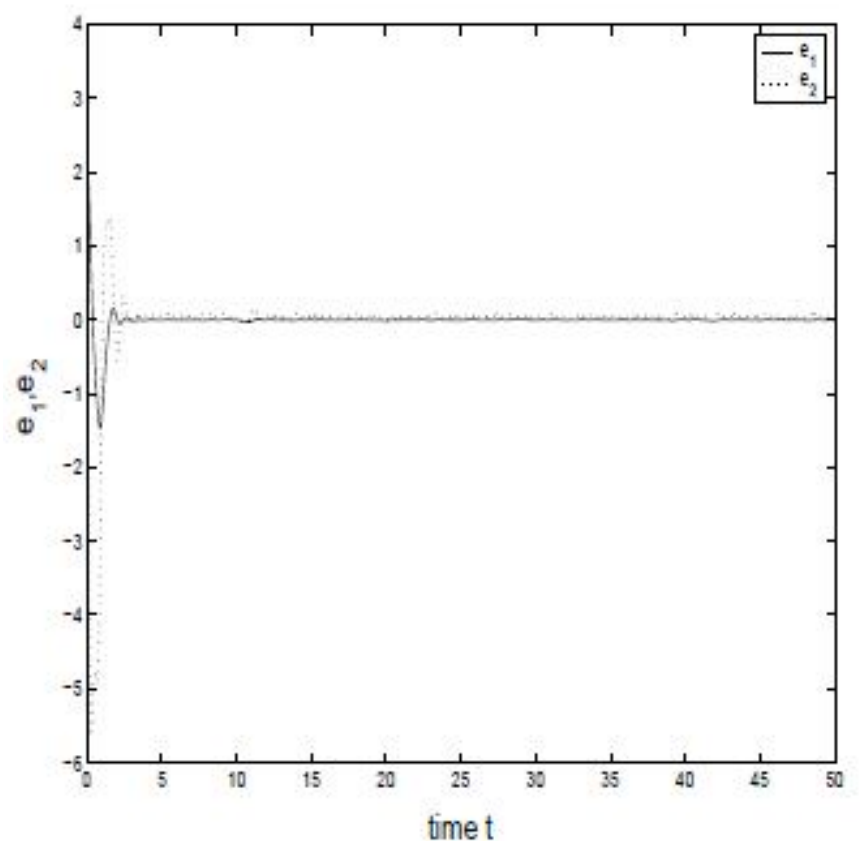

Fig. 6. The error system is also asymptotically stable as $\mathrm{t}$ tends to $\infty$ when synchronization of gyros with unknown parameters is achieved for initial values as inFigure 4 and parameter estimation $(\hat{a}, \hat{b}, \hat{\beta})=(0.4,0.01,0.5)$.

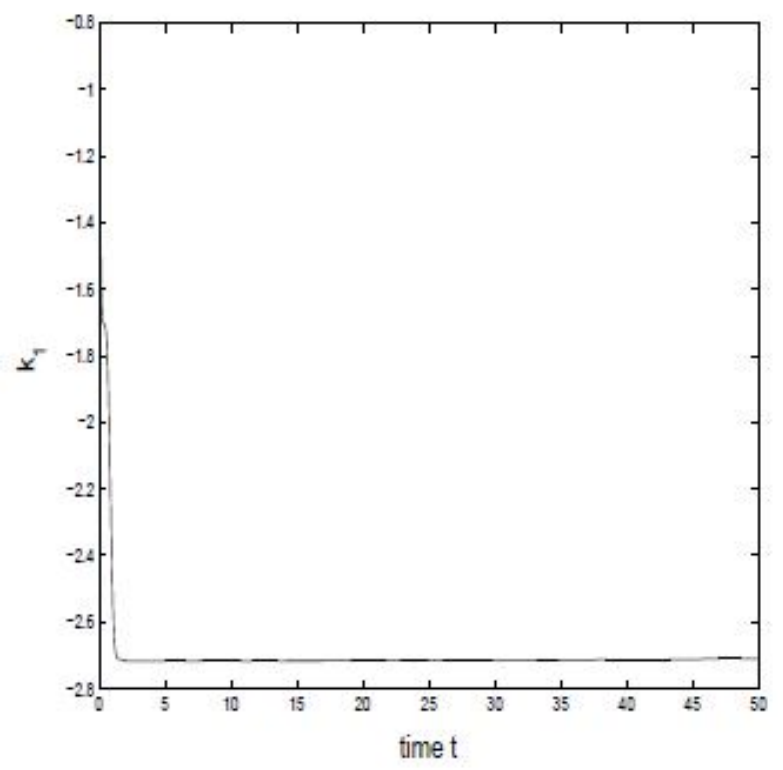

Fig. 7. The feedback gain $k_{1}$ tends to a negative constant as $t$ tends to $\infty$ whensynchronization of gyros with unknown parameters is achieved and parameters areas in Figure 4. 
International Journal of Chaos, Control, Modelling and Simulation (IJCCMS) Vol.2, No.2, June 2013
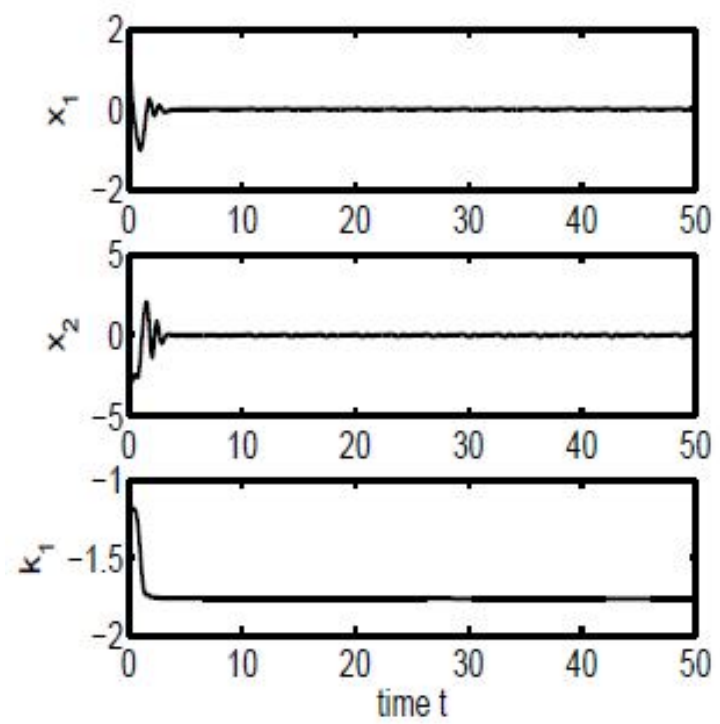

Fig. 8. The gyro system is asymptotically stable to zero as $t \rightarrow \infty$ in thecontrolled state and the feedback gain $k_{1}$ tends to a negative constant as $t$ tends to $\infty$ when noise is added. Initial values are as in Figure 2.
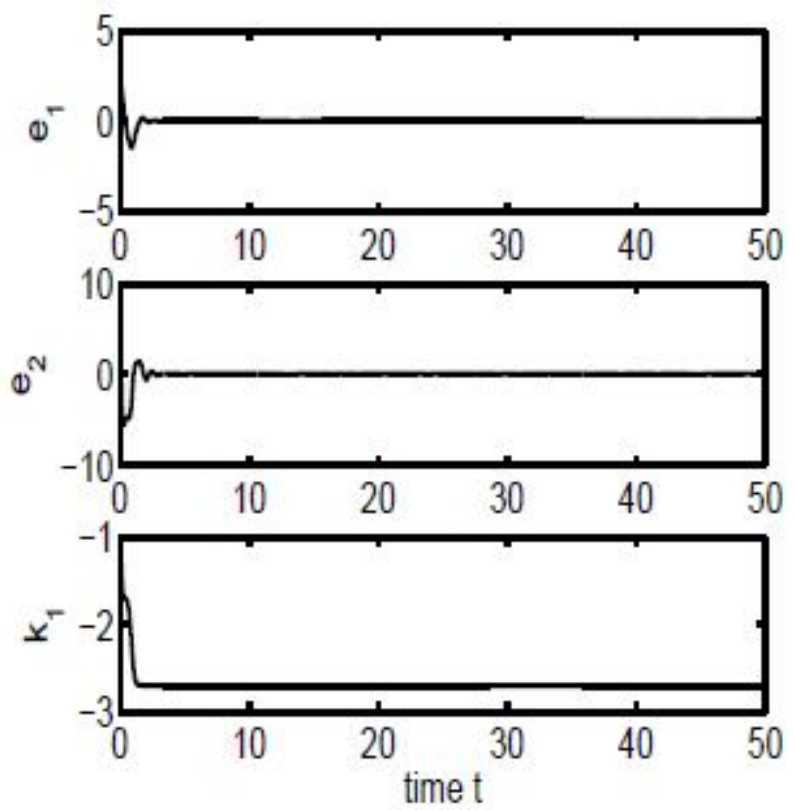

Fig. 9.The case where noise is added to the system. The error system is alsoasymptotically stable ast $\rightarrow \infty$ when synchronization of gyros with differentinitial values as in Figures 4 and 5 is achieved. The feedback gain $k_{1}$ also tends toa negative constant as $t \rightarrow \infty$. 
International Journal of Chaos, Control, Modelling and Simulation (IJCCMS) Vol.2, No.2, June 2013

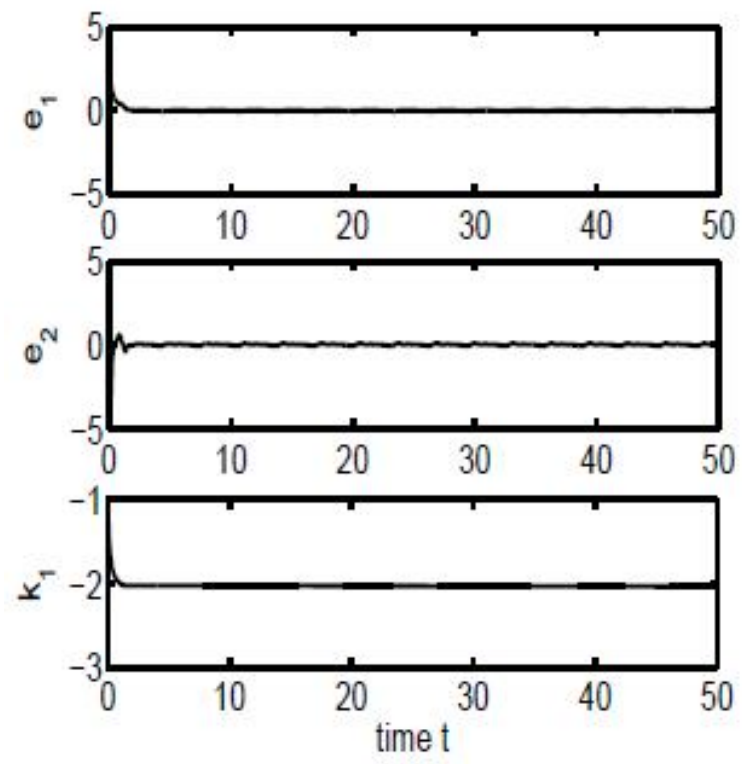

Fig. 10. The error system is also asymptotically stable as $t \rightarrow \infty$ when synchronization of gyros with unknown parameters and noise is added is achieved for initialvalues as in Figures 6 and 7. The feedback gain $k_{1}$ also tends to a negative constantas $t \rightarrow \infty$ when synchronization of the gyros with unknown parameters andnoise is added is achieved. 